\section{Predictors of happiness and satisfaction with life in individuals from the Brazilian general population who use social networks: a cross-sectional study}

\section{Preditores de felicidade e satisfação com a vida em indivíduos da população geral brasileira que utilizam redes sociais: um estudo transversal}

\author{
Predictores de felicidad y satisfacción con la vida \\ en personas de la población brasileña general \\ que usan redes sociales: un estudio transversal
}

\author{
Mayara Goulart de Camargos 1,2 \\ Bianca Sakamoto Ribeiro Paiva 2 \\ Marco Antonio de Oliveira 2 \\ Carla Simone Leite de Almeida 3 \\ Claudia Hofheinz Giacomoni 4 \\ Carlos Eduardo Paiva 2
}

doi: 10.1590/0102-311X00164020

\begin{abstract}
This study aimed to identify possible conditions associated with the perception of happiness and satisfaction with life in a sample of the Brazilian population who use social networks. This was a cross-sectional study with participants recruited online in five regions of Brazil via Facebook and WhatsApp. Data were collected from October 2015 to October 2016. The instruments used were the Pemberton Happiness Index, the Satisfaction with Life Scale, and a questionnaire regarding sociodemographic and clinical characteristics and issues potentially associated with the feeling of happiness. In total, 2,151 participants were included. A total of five variables exerted the greatest influence on higher levels of happiness and satisfaction with life in the multiple linear regression model, in the multiple logistic regression analysis, and in the decision tree model. Being satisfied with financial circumstances, having a positive self-evaluation of health, having frequent family gatherings, engaging in physical activity $\geq 3$ times a week, and having no previous psychological/ psychiatric diagnosis are variables that "seem" to positively influence Brazilians' perception of happiness and satisfaction with life. We identified some predictors of happiness and satisfaction with life, which were mainly related to the social activities and personal satisfaction of the participating individuals. Encouraging people to seek strategies for increasing levels of happiness and life satisfaction based on modifiable variables, such as those found, can be helpful in this context.
\end{abstract}

Happiness; Personal Satisfaction; Social Networking
Correspondence

M. G. Camargos

Hospital de Clínicas da Universidade Federal do Triângulo Mineiro.

Rua Getúlio Guaritá 130, Uberaba, MG 38025-440, Brasil. mayara_camargos@hotmail.com

\footnotetext{
${ }^{1}$ Hospital de Clínicas da Universidade Federal do Triângulo Mineiro, Uberaba, Brasil.

2 Hospital de Câncer de Barretos, Barretos, Brasil.

3 Instituto Federal de Educação, Ciência e Tecnologia de Santa Catarina, Joinville, Brasil.

4 Universidade Federal do Rio Grande do Sul, Porto Alegre, Brasil.
} 


\section{Introduction}

Regardless of how happiness is understood, it is a desire of every human being, and, it can be considered the primary goal of life; humans have tried many paths to achieve it 1 . Several studies have been conducted to understand the pursuit of happiness, many of which have attempted to find "combinations" of variables that may provide people with greater chances of happiness $2,3,4,5,6,7$.

Happiness is a multidimensional and complex construct, mainly because it is a subjective individual experience. Thus, it is challenging to compare data on happiness and its multidimensional measures between different cultures and specific populations 8 . In recent years, happiness has gained increasing attention not only in the academic literature but also in journalistic and political debates 9 .

Happiness is closely associated with emotions, feelings, and moods, whereas satisfaction with life is associated with cognitive assessments and judgments about life, which may include assessments of many areas of life 10. Satisfaction with life is a congruence between the present and an ideal situation, both of which are a reflection of one's own subjective appreciation of one's life 11. Thus, whereas happiness and satisfaction with life are closely related constructs, they must be evaluated as independent variables. In this study, the terms happiness and well-being/subjective well-being will be considered synonyms for better understanding and interpretation.

Social networks have brought intense changes in the way people communicate and interact. However, it is not yet clear whether and how some of these changes, in the form of social interaction, can affect certain aspects of human behavior. Scientific studies still present contradictory results in terms of negative and positive effects of social networks on the mood swings and affections of its users 12 . However, it is a fact that "fake news" negatively affects the mental health of social network users, since they can provoke intense emotional responses, such as anger, fear, anxiety, sadness, impotence, and frustration 13. The significance of the impact of social networks on Brazilian public health is evident considering that $70 \%$ of Brazilians have access to the Internet 14,15 and that Brazil is ranked second in terms of time spent per day on the Internet (approximately 9 hours and 29 minutes per day), with $40 \%$ of this time being spent on social media 16 .

Several studies have studied happiness (and its related constructs) in the search for associated factors, especially those that can be modified, both individually and collectively. Several conditions have been reported to be associated with the perception of happiness. However, besides the predisposing genetic conditions, the sociocultural factors, which are different between the populations, are probably modifiers of the "recipe" for happiness. In Brazil, a large country of multicultural traditions, the rates of happiness and satisfaction with life had not yet been measured within a global context. Thus, the factors that would most significantly influence the perception of happiness of Brazilians were unknown. This study was justified by this lack of scientific literature.

This study was conducted in the context of popular dissatisfaction in Brazil. The hypothesis of the study was that - despite existing in a context wherein people often use social networks for posting about dissatisfaction with violence and corruption - the variables associated with happiness and satisfaction with life are individual and related to healthy social interactions and simple everyday situations (such as leisure activities and contact with nature). Therefore, this study sought to identify possible conditions associated with the perception of happiness and satisfaction with life in a sample of individuals from the Brazilian population who use social networks.

\section{Methods}

\section{Study design}

This was a cross-sectional study performed with convenience sampling. The electronic tool SurveyMonkey (https://www.surveymonkey.com/) was used to collect data. 


\section{Study participants}

Individuals from the Brazilian population who had an account on the Facebook social network and/ or used the WhatsApp software application were included.

\section{Study site}

Data were collected from October 2015 to October 2016. Participants from the five regions of Brazil were recruited online through the Facebook and the WhatsApp. The survey was administered using the SurveyMonkey platform.

\section{Study recruitment strategy}

\section{- Via Facebook}

For the application of the instruments of data collection through the Facebook, an online program called SurveyMonkey was legally acquired by registration on the site https://pt.surveymonkey.com.

For data collection via Facebook, three different methodologies were used. These are described as follows:

(i) Methodology 1: the authors used their personal Facebook pages to share the research post.

(ii) Methodology 2: municipalities were selected according to the demographic and Human Development Index profile of each Brazilian state. To promote the research, we created Facebook pages specific to the study entitled Happiness Research, Happiness Research II, and Happiness Research III. A total of 100 individuals from each municipality were invited. When identifying one or more of these individuals, first contact was made through an inbox message on the person's page and a friend request.

(iii) Methodology 3: researchers without connections to the researchers of this study in the North, Northeast, Central-West, and South were contacted to serve as "poles" for dissemination of the research by Methodology 1 with the objective of propagating the research and reaching populations outside the Southeast region, ultimately reaching a significant number of cities in Brazil.

\section{- Via WhatsApp}

Some people known by the researchers residing in different regions of the country were contacted through the WhatsApp. Explanatory text about the study was sent together with the research link. Besides being asked to answer to the online questionnaire, they were encouraged to share the study text/link with their WhatsApp contacts.

\section{Eligibility criteria}

The inclusion criteria were as follows: Brazilian nationality, dwelling in a Brazilian municipality, a Facebook account and/or use of the WhatsApp.

The only exclusion criterion was individuals younger than 18 years.

\section{Sample size calculation}

The sample size calculation considered an a priori coefficient of determination (effect size) of $\mathrm{R}^{2}=$ 0.01 in a multiple linear regression model with seven predictors and an $\alpha=0.05$ level of significance (or type I error), and 95\% a priori statistical power. In Power Analysis and Sample Size (PASS, 2002 version; https://www.ncss.com/software/pass/), these values yielded a sample size of 2,191 subjects. 


\section{Ethical aspects}

Participants read the informed consent form online before signing it and authorizing their voluntary participation. The study was approved by the Research Ethics Committee of the Barretos Cancer Hospital (opinion n. 1,098,789; CAAE 45007215.0.0000.5437). The study was conducted in accordance with the Declaration of Helsinki.

\section{Data collection instruments}

\section{- Questionnaire of sociodemographic and clinical characteristics and issues potentially associated with the feeling of happiness}

For the development of this questionnaire, the items were defined after meetings among researchers from the Palliative Care and Quality of Life Research Group (GPQual) and were based on a literature review and discussions about potential factors related to happiness. Before proceeding to the main data collection, the first 50 individuals who completed the questionnaire had their answers checked to assess accuracy, to determine the frequency of missing items, and to verify the functioning of SurveyMonkey. The questionnaire included sociodemographic characteristics, such as age, sex, marital status, religion, and region, as well as clinical characteristics, such as personal perception of health and previously diagnosed health problems. Several items addressed issues potentially associated with the perception of happiness (Supplementary Material; http://cadernos.ensp.fiocruz.br/static//arquivo/ suppl-e00164020_9472.pdf).

\section{- Pemberton Happiness Index}

The Pemberton Happiness Index (PHI) is composed of 11 items related to different areas of remembered well-being (general; hedonic; eudaimonic; and social well-being) and 10 items related to recently experienced well-being (previous day events). The items are answered on a Likert scale, and the higher the scores, the greater the happiness. The sum of the scores produces a combined well-being index (PHI-total) 17. The Portuguese version is valid and reliable to use with the Brazilian population through online surveys 18. In this study, the PHI-remembered score (PHI-r), with a cut-off score of 7 , was used to define happiness, according to a validation study in Brazil 18. Cronbach's $\alpha$ value was 0.877 .

\section{- Satisfaction with Life Scale}

This instrument consists of five items that evaluate a cognitive component of life satisfaction, and participants answer it based on a 7-point scale, ranging from 1 (totally disagree) to 7 (totally agree). In the Brazilian validation, the scale presented a Cronbach's $\alpha$ value of 0.8919 . It is the most widely used scale for assessing overall satisfaction with life and has been implemented in several languages and cultures, providing good psychometric indexes 20,21. In this study, Cronbach's $\alpha$ value was 0.873 .

\section{- Study endpoints}

Using the PHI-r, the happiness score was analyzed as both a continuous variable (for the linear regression analysis) and a categorical variable (< or $\geq 7$ for the logistic regression analysis).

Measuring with the Satisfaction with Life Scale (SWLS), the satisfaction with life score was analyzed as a continuous variable (for the linear regression analysis).

\section{Statistical analysis}

The data are described using absolute and relative frequencies. The normality of the data was tested using the Kolmogorov-Smirnov test. The Mann-Whitney or Kruskal-Wallis chi-square and Fisher's exact tests were used to analyze the relationship of the instruments with the variables (univariate 
analysis) and to compare the scores between the groups. For the multivariate analysis, logistic (dichotomous independent variables) and linear (continuous independent variables) regression models were applied. A significance level of $5 \%$ was adopted for the tests, and the analyses were performed using SPSS, version 21.0 (https://www.ibm.com/).

To create decision rules for discriminating between the two groups (PHI-r $\geq 7$ vs. PHI-r $<7$ ), we fitted a model using the decision tree technique by the CHAID method 22.

\section{Results}

The rate of incomplete surveys was $16.1 \%(\mathrm{n}=433)$. The study included 2,151 participants representing the general Brazilian population. The majority were female ( $\mathrm{n}=1,672 ; 77.7 \%$ ), white ( $\mathrm{n}=1,509$; $70.2 \%)$, aged 18-29 years $(\mathrm{n}=940 ; 43.7 \%)$, and married $(\mathrm{n}=1,020 ; 47.4 \%)$, with more than 11 years of schooling ( $\mathrm{n}=1,891 ; 87.9 \%)$. The majority resided in the Southeast region of the country ( $\mathrm{n}=989$; $46 \%)$ and lived in urban areas $(\mathrm{n}=2,096 ; 97.4 \%)$. Table 1 shows the sociodemographic characteristics of the participants.

Univariate analyses were performed for each of the instruments used (PHI and SWLS). Variables with p-value $<0.05$ were included in the linear regression and multiple logistic models, which were adjusted for age, sex, income, and educational level. The detailed results of the univariate analyses are shown in the Tables 2, 3 and 4.

\section{Pemberton Happiness Index}

In the multiple linear regression model (considering the PHI as a continuous variable), being aged between 50 and 59 years $(\beta=0.6 ; \mathrm{p}<0.001)$ and residing in the North $(\beta=0.5 ; \mathrm{p}<0.001)$ and South $(\beta=0.5 ; p<0.001)$ regions of the country were associated with higher happiness scores and more positively self-rated health $(\beta=0.9 ; \mathrm{p}<0.001)$, greater satisfaction with financial circumstances $(\beta=$ $0.7 ; \mathrm{p}<0.001)$, and a greater influence of spiritual/religious life $(\beta=0.4 ; \mathrm{p}<0.001)$. Regarding the daily lives of the participants, more family gatherings $(\beta=0.4 ; \mathrm{p}<0.001)$ and more time for leisure $(\beta=0.5$; $\mathrm{p}<0.001)$, as well as practicing physical activity $\geq 3$ times per week $(\beta=0.4 ; \mathrm{p}<0.001)$, were associated with higher PHI-r scores. On the other hand, a previous psychological/psychiatric diagnosis $(\beta=-0.8$; $\mathrm{p}<0.001)$ and being unemployed $(\beta=-0.8 ; \mathrm{p}<0.001)$ were negatively associated with participants' happiness scores (Table 5).

A multiple logistic regression analysis was also performed with the dichotomized PHI-r (happy vs. not happy) as the independent variable. Individuals aged 50 to 59 years (odds ratio $[\mathrm{OR}]=2.4 ; \mathrm{p}$ $<0.001)$ and residing in the South region $(\mathrm{OR}=1.9 ; \mathrm{p}<0.001)$ of the country were also associated with higher levels of happiness, as well as satisfaction with financial circumstances $(\mathrm{OR}=2.7$; $\mathrm{p}<$ $0.001)$, more positively self-rated health $(\mathrm{OR}=2.3 ; \mathrm{p}<0.001)$, a greater influence of spiritual/religious life $(\mathrm{OR}=1.8 ; \mathrm{p}<0.001)$ and engaging in physical activity $\geq 3$ times per week $(\mathrm{OR}=1.8 ; \mathrm{p}<0.001)$. A previous psychological/psychiatric diagnosis $(\mathrm{OR}=0.4 ; \mathrm{p}<0.001)$ and being female $(\mathrm{OR}=0.7$; $\mathrm{p}<0.005)$ were negatively associated with happiness scores (Table 6).

\section{Satisfaction with Life Scale}

In the multiple linear regression model, we associated greater satisfaction with life with greater satisfaction with financial circumstances $(\beta=3.5$; $\mathrm{p}<0.001)$, greater happiness with work $(\beta=3.1 ; \mathrm{p}<$ $0.001)$, more positively self-rated health $(\beta=3.0 ; \mathrm{p}<0.001)$, more time for leisure $(\beta=1.6 ; \mathrm{p}<0.001)$, more family gatherings $(\beta=1.3 ; \mathrm{p}<0.001)$, a higher family income $(\beta=1.5 ; \mathrm{p}<0.001)$, a greater spiritual/religious influence $(\beta=1.1 ; \mathrm{p}<0.001)$, engaging in physical activity $\geq 3$ times per week $(\beta=0.6$; $\mathrm{p}=0.024)$, and more volunteer work $(\beta=0.7 ; \mathrm{p}=0.019)$. Being unemployed $(\beta=-2.7 ; \mathrm{p}=0.046)$ and having a previous psychological/psychiatric diagnosis $(\beta=-2.3 ; \mathrm{p}<0.001)$ were negatively associated with the participants' satisfaction with life (Table 7). 
Table 1

Sociodemographic characteristics of the participants $(N=2,151)$.

\begin{tabular}{|c|c|c|}
\hline Characteristics & $\mathbf{n}$ & $\%$ \\
\hline \multicolumn{3}{|l|}{ Sex } \\
\hline Female & 1,672 & 77.7 \\
\hline \multicolumn{3}{|l|}{ Race * } \\
\hline White & 1,509 & 70.2 \\
\hline Latino & 484 & 22.5 \\
\hline Black & 86 & 4.0 \\
\hline Asian & 55 & 2.5 \\
\hline \multicolumn{3}{|l|}{ Age (years) } \\
\hline $18-29$ & 940 & 43.7 \\
\hline $30-39$ & 703 & 32.7 \\
\hline $40-49$ & 288 & 13.4 \\
\hline $50-59$ & 156 & 7.3 \\
\hline $60-69$ & 52 & 2.4 \\
\hline$\geq 70$ & 12 & 0.6 \\
\hline \multicolumn{3}{|l|}{ Marital status } \\
\hline Legally or common-law married & 1,020 & 47.4 \\
\hline Single & 960 & 44.6 \\
\hline Separated or divorced & 129 & 6.0 \\
\hline Widowed & 30 & 1.4 \\
\hline Other/Do not know & 12 & 0.6 \\
\hline \multicolumn{3}{|l|}{ Region of residence } \\
\hline Southeast & 989 & 46.0 \\
\hline South & 566 & 26.3 \\
\hline Northeast & 240 & 11.2 \\
\hline Central-West & 182 & 8.5 \\
\hline North & 174 & 8.1 \\
\hline \multicolumn{3}{|l|}{ Type of residence } \\
\hline Urban & 2,096 & 97.4 \\
\hline \multicolumn{3}{|l|}{ Schooling level (years) ** } \\
\hline$>11$ & 1,891 & 87.9 \\
\hline $8-11$ & 223 & 10.4 \\
\hline$<8$ & 35 & 1.6 \\
\hline \multicolumn{3}{|l|}{ Current professional activity } \\
\hline Yes & 2,068 & 96.1 \\
\hline \multicolumn{3}{|c|}{ Family income (Brazilian minimum wage) } \\
\hline$\geq 4$ & 1,716 & 79.8 \\
\hline \multicolumn{3}{|l|}{ Religion } \\
\hline Catholic & 1,073 & 49.9 \\
\hline Spiritist & 394 & 18.3 \\
\hline Evangelical & 340 & 15.8 \\
\hline No formal religion & 313 & 14.6 \\
\hline Other & 25 & 1.2 \\
\hline Atheist/Agnostic & 6 & 0.3 \\
\hline
\end{tabular}

* Missing: $\mathrm{n}=17(0.8 \%)$;

** Missing: $\mathrm{n}=2(0.1 \%)$. 
Table 2

Univariate analysis for the evaluation of characteristics associated with satisfaction with life measured by the Satisfaction With Life Scale (SWLS) $(\mathrm{N}=2,151)$.

\begin{tabular}{|c|c|c|}
\hline Variables & Median (P25-P75) & p-value \\
\hline Sex & & 0.335 \\
\hline Male & $26(20-29)$ & \\
\hline Female & $26(21-30)$ & \\
\hline Race & & 0.044 \\
\hline White & $26(21-30)$ & \\
\hline Black & $25(20-29)$ & \\
\hline Latino & $25(19.5-29)$ & \\
\hline Asian & $27(20-30)$ & \\
\hline Age (years) & & 0.001 \\
\hline $18-29$ & $26(20-29)$ & \\
\hline $30-39$ & $26(21-30)$ & \\
\hline $40-49$ & $27(20.5-30)$ & \\
\hline $50-59$ & $28(23-31)$ & \\
\hline $60-69$ & $27(23.5-30)$ & \\
\hline$\geq 70$ & $29(26-32)$ & \\
\hline Marital status & & $<0.001$ \\
\hline Legally or common-law married & $27(23-30)$ & \\
\hline Widowed & $25(20-29)$ & \\
\hline Separated or divorced & $22(16-28)$ & \\
\hline Single & $25(20-29)$ & \\
\hline Educational level (years) & & 0.014 \\
\hline$<8$ & $25(16-29)$ & \\
\hline $8-11$ & $24(18-30)$ & \\
\hline$>11$ & $26(21-30)$ & \\
\hline Family income (Brazilian minimum wage) & & $<0.001$ \\
\hline$\leq 3.9$ & $23(16-28)$ & \\
\hline$\geq 4$ & $27(22-30)$ & \\
\hline Satisfaction with financial issues & & $<0.001$ \\
\hline Little * & $24(18-28)$ & \\
\hline Much ** & $29(26-32)$ & \\
\hline Current professional activity & & $<0.001$ \\
\hline Yes & $26(21-30)$ & \\
\hline No & $21(14-28)$ & \\
\hline Region of residence & & 0.293 \\
\hline Southeast & $26(21-30)$ & \\
\hline North & $26(21-29)$ & \\
\hline Northeast & $25(19-30)$ & \\
\hline Central-West & $26(19-30)$ & \\
\hline South & $26(21-30)$ & \\
\hline Type of residence & & 0.203 \\
\hline Urban & $26(21-30)$ & \\
\hline Rural & $27(23-30)$ & \\
\hline Government funding program & & $<0.001$ \\
\hline No & $26(21-30)$ & \\
\hline Yes & $25(20-29)$ & \\
\hline
\end{tabular}

(continues) 
Table 2 (continued)

\begin{tabular}{|c|c|c|}
\hline Variables & Median (P25-P75) & p-value \\
\hline Religion & & $<0.001$ \\
\hline Catholic & $27(22-30)$ & \\
\hline Evangelical & $26(20-29)$ & \\
\hline Spiritist & $26(21-30)$ & \\
\hline Other & $26(18-31)$ & \\
\hline Atheist/Agnostic/No formal religion & $25(19-29)$ & \\
\hline Volunteer work & & $<0.001$ \\
\hline No & $26(20-30)$ & \\
\hline Yes & $27(22-30)$ & \\
\hline Voluntary financial donation & & $<0.001$ \\
\hline No & $25(20-29)$ & \\
\hline Yes & $28(23-30)$ & \\
\hline Pet & & 0.339 \\
\hline No & $26(21-30)$ & \\
\hline Yes & $26(20-30)$ & \\
\hline Current health problem & & $<0.001$ \\
\hline Yes & $25(19-29)$ & \\
\hline No & $27(22-30)$ & \\
\hline Previous psychological/psychiatric diagnosis & & $<0.001$ \\
\hline No & $26(21-30)$ & \\
\hline Yes & $22(15-25)$ & \\
\hline Influence of religious or spiritual life on happiness & & $<0.001$ \\
\hline Little * & $25(18-29)$ & \\
\hline Much ** & $27(22-30)$ & \\
\hline Self-assessment of health & & $<0.001$ \\
\hline $\mathrm{Bad} * * *$ & $20(15-26)$ & \\
\hline Good \# & $27(22-30)$ & \\
\hline Frequency of family gatherings & & $<0.001$ \\
\hline Little * & $24(18-28)$ & \\
\hline Much \#\# & $28(23-30)$ & \\
\hline Contact with nature & & $<0.001$ \\
\hline Little * & $26(20-29)$ & \\
\hline Much \#\# & $28(23-31)$ & \\
\hline Physical activity (times per week) & & $<0.001$ \\
\hline Don't practice & $25(18-29)$ & \\
\hline $1-2$ & $26(21-30)$ & \\
\hline 3 or more & $28(23-30)$ & \\
\hline Leisure time & & $<0.001$ \\
\hline Little * & $25(19-29)$ & \\
\hline Much ** & $29(24-31)$ & \\
\hline Feeling of happiness with the professional activity & & $<0.001$ \\
\hline Little * & $23(17-27)$ & \\
\hline Much ** & $28(24-31)$ & \\
\hline
\end{tabular}

* No/Very little/More or less;

** Very much/Extreme;

*** Very poor/Poor/Neither poor nor good;

\# Good/Very good;

\#\# Often/Always. 
Table 3

Univariate analysis for the evaluation of happiness-associated characteristics measured by the Pemberton Happiness Index (PHI) as a continuous variable $(\mathrm{N}=2,151)$.

\begin{tabular}{|c|c|c|}
\hline Variables & Median (P25-P75) & p-value \\
\hline Sex & & 0.256 \\
\hline Male & $7.64(6.18-8.45)$ & \\
\hline Female & $7.45(5.82-8.45)$ & \\
\hline Age (years) & & $<0.001$ \\
\hline $18-29$ & $7.27(5.64-8.27)$ & \\
\hline $30-39$ & $7.55(5.91-8.45)$ & \\
\hline $40-49$ & $7.82(5.82-8.59)$ & \\
\hline $50-59$ & $8.09(7.09-9.09)$ & \\
\hline $60-69$ & $8.18(7.00-8.91)$ & \\
\hline$\geq 70$ & $8.32(6.73-9.41)$ & \\
\hline Marital status & & $<0.001$ \\
\hline Legally or common-law married & $7.73(6.09-8.59)$ & \\
\hline Widowed & $7.68(5.73-8.91)$ & \\
\hline Separated or divorced & $7.64(5.55-8.36)$ & \\
\hline Single & $7.27(5.73-8.27)$ & \\
\hline Family income (Brazilian minimum wage) & & $<0.001$ \\
\hline$\leq 3.9$ & $7.09(5.36-8.27)$ & \\
\hline$\geq 4$ & $7.64(6.09-8.45)$ & \\
\hline Current professional activity & & $<0.001$ \\
\hline Yes & $7.55(6.00-8.45)$ & \\
\hline No & $6.18(4.00-8.00)$ & \\
\hline Region of residence & & 0.011 \\
\hline Southeast & $7.36(5.45-8.45)$ & \\
\hline North & $7.55(6.18-8.55)$ & \\
\hline Northeast & $7.55(6.05-8.50)$ & \\
\hline Central-West & $7.27(5.27-8.45)$ & \\
\hline South & $7.64(6.36-8.55)$ & \\
\hline Volunteer work & & 0.002 \\
\hline No & $7.45(5.82-8.45)$ & \\
\hline Yes & $7.82(6.18-8.64)$ & \\
\hline Voluntary financial donation & & $<0.001$ \\
\hline No & $7.27(5.64-8.36)$ & \\
\hline Yes & $7.91(6.45-8.73)$ & \\
\hline Pet & & 0.497 \\
\hline No & $7.55(6.09-8.45)$ & \\
\hline Yes & $7.55(5.77-8.45)$ & \\
\hline Current health problem & & $<0.001$ \\
\hline Yes & $7.18(5.36-8.27)$ & \\
\hline No & $7.73(6.27-8.55)$ & \\
\hline Previous psychological/psychiatric diagnosis & & $<0.001$ \\
\hline No & $7.55(6.00-8.45)$ & \\
\hline Yes & $5.50(3.09-7.55)$ & \\
\hline Influence of religious or spiritual life on happiness & & $<0.001$ \\
\hline Little * & $7.00(5.18-8.09)$ & \\
\hline Much ** & $7.82(6.36-8.64)$ & \\
\hline
\end{tabular}

(continues) 
Table 3 (continued)

\begin{tabular}{|c|c|c|}
\hline Variables & Median (P25-P75) & p-value \\
\hline Self-assessment of health & & $<0.001$ \\
\hline $\mathrm{Bad} * * *$ & $5.91(4.00-7.55)$ & \\
\hline Good\# & $7.73(6.27-8.55)$ & \\
\hline Frequency of family gatherings & & $<0.001$ \\
\hline Little * & $6.91(5.18-8.00)$ & \\
\hline Much \#\# & $7.91(6.45-8.73)$ & \\
\hline Contact with nature & & $<0.001$ \\
\hline Little * & $7.36(5.73-8.36)$ & \\
\hline Much \#\# & $8.09(6.91-8.82)$ & \\
\hline Physical activity (times per week) & & $<0.001$ \\
\hline Don't practice & $7.09(5.27-8.27)$ & \\
\hline $1-2$ & $7.36(5.91-8.36)$ & \\
\hline 3 or more & $8.00(6.73-8.73)$ & \\
\hline Leisure time & & $<0.001$ \\
\hline Little * & $7.18(5.55-8.18)$ & \\
\hline Much ** & $8.23(6.91-8.91)$ & \\
\hline Satisfaction with financial issues & & $<0.001$ \\
\hline Little * & $7.00(5.27-8.09)$ & \\
\hline Much ** & $8.27(7.27-8.91)$ & \\
\hline
\end{tabular}

* No/Very little/More or less;

** Very much/Extreme;

*** Very poor/Poor/Neither poor nor good;

\# Good/Very good:

\#\# Often/Always.

\section{Table 4}

Univariate analysis for the evaluation of happiness associated characteristics measured by Pemberton Happiness Index (PHI) dichotomized $(\mathrm{N}=2,151)$.

\begin{tabular}{lccc}
\hline Variables & $\begin{array}{c}\text { Unhappy } \\
(\mathbf{P H I}-\mathbf{r}<\mathbf{7}) \\
\mathbf{n}(\%)\end{array}$ & $\begin{array}{c}\text { Happy } \\
(\mathbf{P H I}-\mathbf{r} \geq \mathbf{7}) \\
\mathbf{n}(\%)\end{array}$ & p-value \\
\hline Sex & & & \\
Male & $169(20.1)$ & $310(23.6)$ & \\
Female & $671(79.9)$ & $1,001(76.4)$ & * \\
Race & & & \\
White & $591(70.9)$ & $918(70.6)$ & \\
Black & $31(3.7)$ & $55(42.2)$ & \\
Latino & $191(22.9)$ & $293(22.5)$ & \\
Asian & $21(2.5)$ & $34(2.6)$ & \\
Age (years) & & & \\
18-29 & $418(49.8)$ & $522(39.8)$ & \\
$30-39$ & $276(32.9)$ & $427(32.6)$ & \\
$40-49$ & $95(11.3)$ & $193(14.7)$ & \\
$50-59$ & $35(4.2)$ & $121(9.2)$ & \\
$60-69$ & $13(1.5)$ & $39(3.0)$ & \\
$\geq 70$ & $3(0.4)$ & $9(0.7)$ & \\
\hline
\end{tabular}

(continues) 
Table 4 (continued)

\begin{tabular}{|c|c|c|c|}
\hline Variables & $\begin{array}{c}\text { Unhappy } \\
\text { (PHI-r < 7) } \\
\text { n (\%) }\end{array}$ & $\begin{array}{c}\text { Happy } \\
\text { (PHI-r } \geq 7) \\
\text { n (\%) }\end{array}$ & p-value \\
\hline Marital status & & & $<0.001$ * \\
\hline Legally or common-law married & $352(42.3)$ & $668(51.1)$ & \\
\hline Widowed & $11(1.3)$ & $19(1.5)$ & \\
\hline Separated or divorced & $46(5.5)$ & $83(6.4)$ & \\
\hline Single & $423(50.8)$ & $537(41.1)$ & \\
\hline Educational level (years) & & & 0.245 * \\
\hline$<8$ & $9(1.1)$ & $26(2.0)$ & \\
\hline $8-11$ & $90(10.7)$ & $133(10.2)$ & \\
\hline$>11$ & $741(88.2)$ & $1,150(87.9)$ & \\
\hline Current professional activity & & & $<0.001 *$ \\
\hline Yes & $790(94.0)$ & $1,278(97.5)$ & \\
\hline No & $50(6.0)$ & $33(2.5)$ & \\
\hline Region of residence & & & 0.003 * \\
\hline Southeast & $426(50.7)$ & $563(42.9)$ & \\
\hline North & $64(7.6)$ & $110(8.4)$ & \\
\hline Northeast & $91(10.8)$ & $149(11.4)$ & \\
\hline Central-West & $72(8.6)$ & $110(8.4)$ & \\
\hline South & $187(22.3)$ & 379 (28.9) & \\
\hline Type of residence & & & 0.263 * \\
\hline Urban & $823(98.0)$ & $1,273(97.1)$ & \\
\hline Rural & $17(2.0)$ & $38(2.9)$ & \\
\hline Family income (Brazilian minimum wage) & & & $<0.001$ * \\
\hline$\leq 3.9$ & $212(25.2)$ & $223(17.0)$ & \\
\hline$\geq 4$ & $628(74.8)$ & $1,088(83.0)$ & \\
\hline Government aid & & & 0.762 * \\
\hline No & $704(83.8)$ & $1,106(84.4)$ & \\
\hline Yes & $136(16.2)$ & $205(15.6)$ & \\
\hline Disability retirement & & & 0.318 * \\
\hline No & 831 (98.9) & $1,303(99.4)$ & \\
\hline Yes & $9(1.1)$ & $8(0.6)$ & \\
\hline Sickness aid & & & 0.220 * \\
\hline No & $836(99.5)$ & $1,298(99.0)$ & \\
\hline Yes & $4(0.5)$ & $13(1.0)$ & \\
\hline Government funding program & & & 0.035 * \\
\hline No & $828(98.6)$ & $1,304(99.5)$ & \\
\hline Yes & $12(1.4)$ & $07(0.5)$ & \\
\hline Religion & & & 0.061 * \\
\hline Catholic & $404(48.1)$ & $669(51.0)$ & \\
\hline Evangelical & $130(15.5)$ & $210(16.0)$ & \\
\hline Spiritist & $147(17.5)$ & $247(18.8)$ & \\
\hline Other & $11(1.3)$ & $14(1.1)$ & \\
\hline Atheist/Agnostic/No formal religion & $148(17.6)$ & $171(13.0)$ & \\
\hline Volunteer work & & & 0.011 * \\
\hline No & 667 (79.4) & $977(74.5)$ & \\
\hline Yes & $173(20.6)$ & $334(25.5)$ & \\
\hline Voluntary financial donation & & & $<0.001$ * \\
\hline No & $623(74.2)$ & $828(63.2)$ & \\
\hline Yes & $217(25.8)$ & $483(36.8)$ & \\
\hline
\end{tabular}

(continues) 
Table 4 (continued)

\begin{tabular}{|c|c|c|c|}
\hline Variables & $\begin{array}{c}\text { Unhappy } \\
\text { (PHI-r < 7) } \\
\text { n (\%) }\end{array}$ & $\begin{array}{c}\text { Happy } \\
\text { (PHI-r } \geq 7) \\
\text { n (\%) }\end{array}$ & p-value \\
\hline Pet & & & 0.322 \\
\hline No & $329(39.2)$ & $542(41.3)$ & \\
\hline Yes & $511(60.8)$ & 769 (58.7) & \\
\hline Leisure time & & & $<0.001$ * \\
\hline Little ** & $664(79.0)$ & 809 (61.7) & \\
\hline Much *** & $176(21.0)$ & $502(38.3)$ & \\
\hline Self-assessment of health & & & $<0.001$ * \\
\hline Bad \# & $203(24.2)$ & $116(8.8)$ & \\
\hline Good \#\# & $637(75.8)$ & $1,195(91.2)$ & \\
\hline Frequency of family gatherings & & & $<0.001$ * \\
\hline Little ** & $438(52.1)$ & $436(33.3)$ & \\
\hline Much \#\#\# & 402 (47.9) & 875 (66.7) & \\
\hline Influence of religious or spiritual life on happiness & & & $<0.001$ * \\
\hline Little ** & $405(48.2)$ & $410(31.3)$ & \\
\hline Much *** & $435(51.8)$ & $901(68.7)$ & \\
\hline Contact with nature & & & $<0.001$ * \\
\hline Little ** & 747 (88.9) & $1,041(79.4)$ & \\
\hline Much \#\#\# & $93(11.1)$ & $270(20.6)$ & \\
\hline Physical activity (times per week) & & & $<0.001$ * \\
\hline Don't practice & $382(45.5)$ & $441(33.6)$ & \\
\hline $1-2$ & $238(28.3)$ & $329(25.1)$ & \\
\hline 3 or more & $220(26.2)$ & $541(41.3)$ & \\
\hline Feeling of happiness with the professional activity & & & $<0.001$ * \\
\hline Little ** & $423(56.2)$ & $295(24.1)$ & \\
\hline Much *** & $330(43.8)$ & $931(75.9)$ & \\
\hline Satisfaction with financial issues & & & $<0.001$ * \\
\hline Little ** & $676(80.5)$ & $708(54.0)$ & \\
\hline Much *** & $164(19.5)$ & $603(46.0)$ & \\
\hline Current health problem & & & $<0.001$ * \\
\hline Yes & $433(51.5)$ & $528(40.3)$ & \\
\hline No & $407(48.5)$ & $783(59.7)$ & \\
\hline Previous psychological/psychiatric diagnosis & & & $<0.001$ * \\
\hline No & 804 (95.7) & 1,297 (98.9) & \\
\hline Yes & $36(4.3)$ & $14(1.1)$ & \\
\hline
\end{tabular}

PHI-r: PHI-remembered score.

* Chi-square test;

** No/Very little/More or less;

*** Very much/Extreme;

\# Very poor/Poor/Neither poor nor good;

\#\# Good/Very good;

\#\#\# Often/Always. 
Table 5

Multiple linear regression to evaluate the characteristics related with happiness measured by the Pemberton Happiness Index (PHI) (N=2,151).

\begin{tabular}{|c|c|c|c|}
\hline Characteristics & $\beta(\mathrm{SE})$ * & $95 \% \mathrm{Cl}$ & p-value \\
\hline Constant & $4.9(0.1)$ & $4.7 ; 5.2$ & $<0.001$ \\
\hline \multicolumn{4}{|l|}{ Age (years) } \\
\hline $18-29$ & - & - & - \\
\hline $30-39$ & $0.1(0.1)$ & $-0.1 ; 0.2$ & 0.315 \\
\hline $40-49$ & $0.3(0.1)$ & $0.0 ; 0.5$ & 0.015 \\
\hline $50-59$ & $0.6(0.1)$ & $0.3 ; 0.9$ & $<0.001$ \\
\hline $60-69$ & $0.6(0.2)$ & $0.1 ; 1.0$ & 0.019 \\
\hline$\geq 70$ & $0.5(0.5)$ & $-0.5 ; 1.4$ & 0.312 \\
\hline \multicolumn{4}{|l|}{ Region of residence } \\
\hline Southeast & - & - & - \\
\hline Central-West & $0.0(0.1)$ & $-0.3 ; 0.3$ & 0.986 \\
\hline Northeast & $0.3(0.1)$ & $0.0 ; 0.5$ & 0.021 \\
\hline North & $0.5(0.1)$ & $0.3 ; 0.8$ & $<0.001$ \\
\hline South & $0.5(0.1)$ & $0.3 ; 0.6$ & $<0.001$ \\
\hline \multicolumn{4}{|l|}{ Employment } \\
\hline Yes & - & - & - \\
\hline No & $-0.8(0.2)$ & $-1.2 ;-0.4$ & $<0.001$ \\
\hline \multicolumn{4}{|c|}{ Satisfaction with financial circumstances } \\
\hline Very much ** & - & - & - \\
\hline Little *** & $0.7(0.1)$ & $0.6 ; 0.9$ & $<0.001$ \\
\hline \multicolumn{4}{|l|}{ Self-assessed health } \\
\hline Poor \# & - & - & - \\
\hline Good \#\# & $0.9(0.1)$ & $1.4 ; 2.5$ & $<0.001$ \\
\hline \multicolumn{4}{|c|}{ Prior psychological/psychiatric diagnosis } \\
\hline No & - & - & - \\
\hline Yes & $-0.8(0.1)$ & $-0.9 ;-0.6$ & $<0.001$ \\
\hline \multicolumn{4}{|c|}{ Frequency of family gatherings } \\
\hline Infrequent $* \star *$ & - & - & - \\
\hline Very frequent \#\#\# & $0.4(0.1)$ & $0.3 ; 0.6$ & $<0.001$ \\
\hline \multicolumn{4}{|c|}{ Influence of religious or spiritual life on happiness } \\
\hline Little *** & - & - & - \\
\hline Very much ** & $0.4(0.1)$ & $0.3 ; 0.6$ & $<0.001$ \\
\hline \multicolumn{4}{|l|}{ Leisure time } \\
\hline Little *** & - & - & - \\
\hline Very much ** & $0.5(0.1)$ & $0.3 ; 0.6$ & $<0.001$ \\
\hline \multicolumn{4}{|c|}{ Physical activity (times per week) } \\
\hline Don't practice & - & - & - \\
\hline $1-2$ & $0.1(0.1)$ & $-0.1 ; 0.3$ & 0.403 \\
\hline 3 or more & $0.4(0.1)$ & $0.2 ; 0.6$ & $<0.001$ \\
\hline
\end{tabular}

95\%Cl: 95\% confidence interval; SE: standard error.

Note: model adjusted for the following variables: age, sex, family income, and educational level $\left(R^{2}=0.266\right)$.

* Coefficient estimated by linear model;

** Very much/Extreme;

$\star \star \star *$ No/Very little/More or less;

\# Very poor/Poor/Neither poor nor good;

\#\# Good/Very good;

\#\#\# Often/Always. 
Table 6

Logistic regression to evaluate the characteristics associated with happiness measured by the dichotomized Pemberton Happiness Index $(\mathrm{PHI})(\mathrm{N}=2,151)$.

\begin{tabular}{|c|c|c|c|}
\hline Characteristics & OR & $95 \% \mathrm{Cl}$ & p-value \\
\hline Constant & 0.3 & - & $<0.001$ \\
\hline \multicolumn{4}{|l|}{ Sex } \\
\hline Male & - & - & - \\
\hline Female & 0.7 & $0.5 ; 0.9$ & 0.005 \\
\hline \multicolumn{4}{|l|}{ Age (years) } \\
\hline $18-29$ & 1.0 (Reference) & & \\
\hline $30-39$ & 1.1 & $0.9 ; 1.3$ & 0.513 \\
\hline $40-49$ & 1.7 & $1.2 ; 2.3$ & 0.002 \\
\hline $50-59$ & 2.4 & $1.6 ; 3.8$ & $<0.001$ \\
\hline $60-69$ & 1.9 & $0.9 ; 4.1$ & 0.057 \\
\hline$\geq 70$ & 1.5 & $0.4 ; 6.0$ & 0.544 \\
\hline \multicolumn{4}{|l|}{ Region of residence } \\
\hline Southeast & 1.0 (Reference) & & \\
\hline North & 1.7 & $1.2 ; 2.5$ & 0.004 \\
\hline Northeast & 1.4 & $1.0 ; 2.0$ & 0.034 \\
\hline Central-West & 1.1 & $0.8 ; 1.6$ & 0.458 \\
\hline South & 1.9 & $1.5 ; 2.4$ & $<0.001$ \\
\hline \multicolumn{4}{|c|}{ Satisfaction with financial circumstances } \\
\hline Little * & - & - & - \\
\hline Very much ** & 2.7 & $2.1 ; 3.4$ & $<0.001$ \\
\hline \multicolumn{4}{|c|}{ Influence of religious or spiritual life on happiness } \\
\hline Little * & - & - & - \\
\hline Very much ** & 1.8 & $1.5 ; 2.2$ & $<0.001$ \\
\hline \multicolumn{4}{|l|}{ Self-assessed health } \\
\hline Poor $* * *$ & - & - & - \\
\hline Good \# & 2.3 & $1.7 ; 3.1$ & $<0.001$ \\
\hline \multicolumn{4}{|c|}{ Prior psychological/psychiatric diagnosis } \\
\hline No & - & - & - \\
\hline Yes & 0.4 & $0.3 ; 0.5$ & $<0.001$ \\
\hline \multicolumn{4}{|c|}{ Frequency of family gatherings } \\
\hline Infrequent * & - & - & - \\
\hline Very frequent \#\# & 1.7 & $1.4 ; 2.1$ & $<0.001$ \\
\hline \multicolumn{4}{|c|}{ Physical activity (times per week) } \\
\hline Don't practice & 1.0 (Reference) & & \\
\hline $1-2$ & 1.0 & $0.8 ; 1.3$ & 0.833 \\
\hline 3 or more & 1.8 & $1.4 ; 2.2$ & $<0.001$ \\
\hline
\end{tabular}

95\% Cl: 95\% confidence interval; OR: odds ratio.

Note: model adjusted for the following variables: age, sex, family income, and educational level $\left(R^{2}=0.267\right)$.

* No/Very little/More or less;

** Very much/Extreme;

*** Very poor/Poor/Neither poor nor good;

\# Good/Very good;

\#\# Often/Always. 
Table 7

Multiple linear regression to evaluate characteristics associated with life satisfaction, measured by the Satisfaction with Life Scale (SWLS) (N = 2,151).

\begin{tabular}{|c|c|c|c|}
\hline Characteristics & $\beta$ (SE) & $95 \% \mathrm{Cl}$ & p-value \\
\hline Constant & $16.1(0.5)$ & $15.1 ; 16.9$ & $<0.001$ \\
\hline \multicolumn{4}{|l|}{ Employed } \\
\hline Yes & - & - & - \\
\hline No & $-2.7(1.4)$ & $-5.4 ;-0.1$ & 0.046 \\
\hline \multicolumn{4}{|c|}{ Family income (Brazilian minimum wage) } \\
\hline$\leq 3.9$ & - & - & - \\
\hline$\geq 4$ & $1.5(0.3)$ & $0.8 ; 2.1$ & $<0.001$ \\
\hline \multicolumn{4}{|l|}{ Volunteer work } \\
\hline No & - & - & - \\
\hline Yes & $0.7(0.3)$ & $0.1 ; 1.2$ & 0.019 \\
\hline \multicolumn{4}{|c|}{ Satisfaction with financial circumstances } \\
\hline Little * & - & - & - \\
\hline Very much ** & $3.5(0.3)$ & $2.9 ; 4.0$ & $<0.001$ \\
\hline \multicolumn{4}{|l|}{ Self-assessed health } \\
\hline Poor *** & - & - & - \\
\hline Good \# & $3.0(0.4)$ & $2.3 ; 3.7$ & $<0.001$ \\
\hline \multicolumn{4}{|c|}{ Prior psychological/psychiatric diagnosis } \\
\hline No & - & - & - \\
\hline Yes & $-2.3(0.3)$ & $-2.9 ;-1.8$ & $<0.001$ \\
\hline \multicolumn{4}{|c|}{ Frequency of family reunions } \\
\hline Infrequent * & - & - & - \\
\hline Very frequent \#\# & $1.3(0.2)$ & $0.8 ; 1.8$ & $<0.001$ \\
\hline \multicolumn{4}{|c|}{ Influence of religious or spiritual life on happiness } \\
\hline Little * & - & - & - \\
\hline Very much ** & $1.1(0.2)$ & $0.6 ; 1.6$ & $<0.001$ \\
\hline \multicolumn{4}{|l|}{ Leisure time } \\
\hline Little * & - & - & - \\
\hline Very much ** & $1.6(0.3)$ & $1.1 ; 2.2$ & $<0.001$ \\
\hline \multicolumn{4}{|l|}{ Happiness with work } \\
\hline Little * & - & - & - \\
\hline Very much ** & $3.1(0.2)$ & $2.6 ; 3.6$ & $<0.001$ \\
\hline \multicolumn{4}{|c|}{ Physical activity (times per week) } \\
\hline Don't practice & - & - & - \\
\hline $1-2$ & $0.3(0.3)$ & $-0.3 ; 0.9$ & 0.376 \\
\hline 3 or more & $0.6(0.3)$ & $0.1 ; 1.2$ & 0.024 \\
\hline
\end{tabular}

95\% Cl: 95\% confidence interval; SE: standard error.

Note: model adjusted for the following variables: age, sex, family income, and educational level $\left(\mathrm{R}^{2}=0.362\right)$.

* No/Very little/More or less;

** Very much/Extreme;

$* \star \star$ Very poor/Poor/Neither poor nor good;

\# Good/Very good;

\#\# Often/Always. 


\section{Decision tree model}

A tool was also generated through the decision tree technique using the CHAID method 22 to discriminate between two groups according to the PHI-r (happy vs. not happy).

Among groups, the decision tree model generated simple decision rules to differ their respective probabilities of correct differentiation. This type of model can be particularly useful for rapid assessment. Among the variables, five remained the most important: satisfaction with financial circumstances, self-assessed health, previous psychological/psychiatric diagnosis, frequency of family gatherings, and engagement in physical activity (Figure 1).

The five important variables that remained in the decision tree model also stood out in the linear regression and logistics analyses, in which everyone had an influence on satisfaction with life (SWLS) and the perception of happiness (PHI-r) (Table 8).

\section{Discussion}

This study aimed to identify possible predictors of the perception of happiness and satisfaction with life in a sample of individuals from the Brazilian population who use social networks. We found a set of variables that positively influence the feeling of happiness and satisfaction with life, including satisfaction with financial circumstances, self-assessed health, previous psychological/psychiatric diagnosis, frequency of family gatherings, and engagement in physical activity.

\section{Main findings}

Regarding the sociodemographic variables, sex and age are noteworthy. Sex is a sociodemographic variable associated with inconsistent findings in the literature regarding happiness since it can be influenced by social origins and the cultural contexts of each country ${ }^{5}$. Some of the differences

Table 8

Influence of variables on happiness and satisfaction with life scores.

\begin{tabular}{|c|c|c|c|c|}
\hline \multirow[t]{2}{*}{ Characteristics } & \multicolumn{3}{|c|}{ Happiness * } & \multirow[t]{2}{*}{ Satisfaction with life ** } \\
\hline & Dichotomous variable *** & Continous variable & Tree \# & \\
\hline Satisfaction with financial circumstances & + & + & + & + \\
\hline Self-assessed health & + & + & + & + \\
\hline Engagement in physical activity & + & + & + & + \\
\hline Frequency of family gatherings & + & + & + & + \\
\hline Prior psychological/psychiatric diagnosis & + & + & + & + \\
\hline Spirituality/religiosity & + & + & - & + \\
\hline Age & + & + & - & - \\
\hline Employment & - & + & - & + \\
\hline Region of residence & + & + & - & - \\
\hline Leisure activities & - & + & - & + \\
\hline Sex & + & - & - & - \\
\hline Family income & - & - & - & + \\
\hline Volunteer work & - & - & - & + \\
\hline Happiness with work & - & - & - & + \\
\hline
\end{tabular}

* Pemberton Happiness Index;

** Satisfaction with Life Scale;

$\star * \star$ Yes/No;

\# Result of the decision tree using PHI-remembered score (PHI-r) as a dichotomous variable. 


\section{Figure 1}

Decision tree model with simple decision rules (considering the studied variables) to discriminate between the groups and their respective probabilities of being considered happy or unhappy.

\begin{tabular}{|c|c|c|c|c|c|c|c|}
\hline & $\begin{array}{l}\text { Unhappy } \\
\text { (PHI-r < 7) }\end{array}$ & $\begin{array}{l}\text { Unhappy } \\
(\mathrm{PHI}-\mathrm{r}<7)\end{array}$ & $\begin{array}{c}\text { Happy } \\
(\mathrm{PHI}-\mathrm{r} \geq 7)\end{array}$ & $\begin{array}{c}\text { Happy } \\
(\mathrm{PHI}-\mathrm{r} \geq 7)\end{array}$ & $\begin{array}{c}\text { Happy } \\
(\mathrm{PHI}-\mathrm{r} \geq 7)\end{array}$ & $\begin{array}{c}\text { Happ } \\
(\mathrm{PHI}-\mathrm{r} \geq 7)\end{array}$ & $\begin{array}{c}\text { Happy } \\
(\mathrm{PHI}-\mathrm{r} \geq 7)\end{array}$ \\
\hline $\begin{array}{l}\text { Satisfaction with financial } \\
\text { circurmstances }\end{array}$ & Little * & Little * & Little * & Little * & $\begin{array}{l}\text { Very } \\
\text { much ** }\end{array}$ & $\begin{array}{l}\text { Very } \\
\text { much ** }\end{array}$ & $\begin{array}{l}\text { Very } \\
\text { much ** }\end{array}$ \\
\hline $\begin{array}{l}\text { Previous psychiatric/ } \\
\text { psychological diagnosis }\end{array}$ & Yes & Yes & No & No & Yes & No & No \\
\hline Self-assessed health & Poor *** & Good \# & 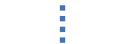 & & & : & \\
\hline Frequency of family gatherings & & & Little * & $\begin{array}{l}\text { Very } \\
\text { frequent }\end{array}$ & & & \\
\hline Physical activity (times per week) & & & & & & $0-2$ & $\geq 3$ \\
\hline $\begin{array}{l}\text { Probability of correction } \\
\text { prediction }\end{array}$ & & & & & & & \\
\hline
\end{tabular}

PHI-r: PHI-remembered score.

* No/Very little/More or less;

** Very much/Extreme;

*** Very poor/Poor/Neither poor nor good;

\# Good/Very good;

\#\# Often/Always.

related to satisfaction with life may be due to the different weights attributed by men and women to different dimensions of life, such as occupational, social, health, or housing factors 23 .

The relationship between age and happiness has also been investigated, and the distribution of happiness levels was depicted as a U-shaped curve 24. This indicates that younger and older adults tend to have higher levels of happiness than middle-aged adults 24 . In this study, more than $75 \%$ of participants were aged from 18 to 39 years. According to the results, individuals aged between 40 and 70 reported being happier than the younger people. Analysis of individuals aged over 70 years was hampered by the small number of participants in this stratum.

In this study, having a job and being happy with it were significant factors for participants' happiness and satisfaction with life. Consistent with this finding, we found positive associations between being employed and happiness indexes 25,26 . Furthermore, job satisfaction has been strongly correlated - among other things - with happiness and satisfaction with life 25. Moreover, the chances of a person reporting happiness may be greater if the individual evaluates the nature of their work positively 25 . This is because, likely for many individuals, work also contributes to the development of identity and because the assessment of work can affect other spheres of life 26. 
Although several studies provide evidence of an association between volunteer work and increased levels of happiness 27 , these effects have greater meaning for older adults than for younger individuals 27,28 , and the positive influences on happiness are greater in those with a lower socioeconomic status 27,29 . Being involved in some type of volunteer activity in this study was associated with satisfaction with life but not with happiness, which may be explained by the fact that most participants were young, with a high educational level and a high income.

Enjoyable leisure activities have been associated with psychosocial and physical measures relevant to health and well-being 30 . In our study, we associated leisure activities with both happiness and satisfaction with life. Besides facilitating moments of relaxation, leisure activities often provide social interactions, which are positively conducive to happiness and satisfaction with life 31,32 . In a Japanese cohort of middle-aged adults, mental health status was significantly associated with leisure activities (cultural activities or sports), especially those involving others, suggesting the importance of social interactions 33 .

Family relations are another form of social interaction, since these are fundamental for providing, among other things, financial and emotional support and, consequently, social and psychological support 34. The search for family harmony is considered a significant purpose of life that is also essential for maintaining the happiness of individuals regardless of culture and age group 34,35. We found evidence of an association between positive family relations and happiness 34 . The frequency of family gatherings (e.g., family lunches or dinners) was positively associated with happiness and life satisfaction and it was also an important item in the decision tree. A previous study associated having companionship during meals with higher happiness scores 35 .

A very widespread issue in Western society is whether "money can buy happiness". Our results show that although family income was associated with higher satisfaction with life, personal satisfaction with the earned money tended to be more relevant, as it was positively associated with happiness and satisfaction with life; it was also one of the items present in the decision tree. How much money the individual earns alone does not predict satisfaction in other areas of life 5,36. Therefore, it is possible to find happy people with very low incomes, which could explain why they experience great satisfaction in other areas of their lives 2,36. However, the existence of a positive relationship between family financial satisfaction and happiness is known ${ }^{6}$. Although money cannot buy happiness, it can, for example, make health care accessible, especially in regions with exacerbated socioeconomic inequalities and scarce healthcare resources 37 . Therefore, the transition from poverty to moderate income is fundamental for a family to meet its basic needs 38 . After meeting basic needs, however, the additional income does not serve deeper needs in a lasting way, at least when it is directed toward acquiring more material goods 39. Experiential purchases (such as vacations, travel, concerts, and meals in restaurants) tend to bring more lasting happiness than material purchases. This is because, compared to material goods, experiences are less prone to hedonic adaptation 40.

We positively associated self-assessed health, also called self-reported health, with happiness $2,3,4,6,34$, even after controlling the results by relevant socioeconomic phenomena $2,9,37$. In our study, self-assessed health seemed to influence happiness and satisfaction with life, and to be a relevant item in the decision tree. What matters in the self-assessment of health, which is a subjective assessment of the individual, is the individual's feeling of being in good or poor health, regardless of the actual number of illnesses present 2,3.

Scientific evidence indicates that the practice of physical activity is also positively correlated with happiness scores 41 , and that individuals are happier at times when they are more physically active 41 . Such momentary happiness could be related to underlying social interactions, since reports of more positive effects when individuals are in social situations ${ }^{42}$. Physical activity is also probably linked to happiness by internal processes, as it provides a revitalizing effect, which can increase the availability of resources for the pursuit of personal goals 42 . In this study, practicing physical activity more than three times a week was associated with happiness and satisfaction with life and was one of the relevant items in the decision tree.

Being happy is not necessarily the opposite of being depressed. In any case, it seems natural to assume that happiness is negatively associated with negative emotional traits 8 , as well as other mental disorders 43 . Average happiness seems to be higher in countries that invest more in mental health, both in absolute terms (more mental health professionals) and relative terms (share of investment in 
mental health care in the total health care budget), especially in developed countries 43 . Our results corroborate those of previous studies showing that a diagnosis of depression or anxiety is associated with a lower level of happiness 7 . Likewise, satisfaction with life is also strongly influenced by mental health 44 .

Spirituality/religiosity was another significant factor of the happiness and satisfaction with life of the participants from the general Brazilian population. The relationship between spirituality/ religiosity and satisfaction with life has been found to be positive 1,6. Scientific evidence suggests that individuals who regularly attend religious institutions build social networks, but that the effect of the social relations arising from such encounters is contingent on the presence of a strong religious identity; moreover, it may occur differences in the experience of happiness and satisfaction with life in different spiritual/religious groups 6 . At the same time, spirituality/religiosity is a way to achieve purpose in life, to improve mental health, to establish well-being, and to gain inner peace, which can lead to happiness 1 .

Scientific publications have demonstrated that a healthy and creative emotional life is a protective factor against disease and plays an important role in the favorable evolution of chronic diseases. The search for well-being and happiness is inherent to the human condition. The identification of conditions associated with a greater perception of happiness can provide subsidies for national public policies aimed at improving the population's living conditions. We still do not know what should be encouraged (or discouraged) for individual happiness, especially in the Brazilian's life. Furthermore, during the data collection period, Brazil was going through a time of political and economic instability. Thus, the results of this study are able to help understand the impact of such a crisis on people's lives.

The work philosophy of every health professional should focus on the humanization of care. Regardless of the profile of potential patients served by them, this professional should see the Brazilian citizen as deserving of adequate health care, respect for individuality, and encouragement for healthy living practices, including the variables associated with happiness and life satisfaction reported in this study (related to healthy social interactions and simple everyday situations). Furthermore, the decision tree model cited is simple and easy to interpret as well as can offer a convenient application in clinical practice to identify possible risks of unhappiness and preventive actions against those risks.

Issues related to happiness have become an important topic for public policy, economics, and psychology in recent years 45. The United Nations (UN) even passed a resolution recognizing the pursuit of happiness as a fundamental human objective. More than an individual desire, the UN establishes the importance of creating public policies for this purpose 46 , encouraging each country to develop measures that reflect its characteristics. For this reason, happiness was considered, contemplated or even incorporated into public policies in several countries.

\section{Limitations of the study}

This study has some limitations. We recruited the participants only through social networks (Facebook and WhatsApp), which can contribute to internet access bias because not all Brazilians have online access. The spontaneous interest in the subject may serve as another selection bias insofar as the individuals were free to participate when receiving the invitation through the social network. The discrepancy in the number of respondents of the female sex is another limitation, besides the inequality in the number of participants by region of the country, although all the Brazilian regions were represented. To minimize potential sampling bias, we adjusted all analyses for age, sex, income, and educational level.

\section{Strengths and practical perspectives}

This study benefits from strengths with regard to its originality - namely, in Brazil, we did not find other study evaluating the specified predictors - and the large number of its participants, all of the same nationality but residing in different regions. Since the relevant predictors was identified, it was possible to stimulate increases in their frequency of occurrence in the daily life of Brazilians in an attempt to raise individual levels of happiness and satisfaction with life. 
In individuals with a history of psychological/psychiatric disorders, therapeutic strategies focused on improving self-perceived health, as well as cognitive adjustment regarding expectations of financial gain (i.e., satisfaction with financial circumstances), may be important for the individual perception of happiness. Encouraging individuals to seek closeness with family members can also be helpful in this context. However, these therapeutic strategies need to be delineated and tested in future studies.

\section{Conclusions}

A set of variables predicted the perception of happiness and satisfaction with life of a sample of individuals from the Brazilian population in general through social networks. Being satisfied with financial circumstances, having a positive perception of one's self-evaluated health, having frequent family gatherings, engaging in physical activity $\geq 3$ times a week, and not having a previous psychological/psychiatric diagnosis are variables that "seem" to influence, in a positive way, the perception of happiness.

The decision tree model is simple and easy to interpret, as well as it can offer a convenient application in clinical practice to identify possible risks for unhappiness and preventive actions against these risks.

\section{Contributors}

M. G. Camargos and C. E. Paiva conceived and designed the study, performed the experiments, analyzed the data, and wrote and revised the manuscript. B. S. R. Paiva conceived and designed the study, performed the experiments, and revised the manuscript. M. A. Oliveira analyzed the data and revised the manuscript. C. S. L. Almeida performed the experiments and revised the manuscript. C. $\mathrm{H}$. Giacomoni wrote and revised the manuscript. All authors approved the final version of the manuscript.

\section{Additional informations}

ORCID: Mayara Goulart de Camargos (0000-00030443-8301); Bianca Sakamoto Ribeiro Paiva (00000002-2711-8346); Marco Antonio de Oliveira (0000-0001-6879-2778); Carla Simone Leite de Almeida (0000-0003-1220-5452); Claudia Hofheinz Giacomoni (0000-0002-9183-8591); Carlos Eduardo Paiva (0000-0002-7934-1451).

\section{Acknowledgments}

The authors thank the researchers Isabel Cristina Belasco and Vanessa Regina Maciel Uzan for help in data collection. This project was supported by the Barretos Cancer Hospital internal research funds (PAIP). 


\section{References}

1. Rizvi MAK, Hossain MZ. Relationship between religious belief and happiness: a systematic literature review. J Relig Health 2017; 56:1561-82.

2. Panadero S, Guillen AI, Vazquez JJ. Happiness on the street: overall happiness among homeless people in Madrid (Spain). Am J Orthopsychiatry 2015 ; 85:324-30.

3. Ramirez de Arellano AB. Calibrating contentment: the metrics of health and happiness. P R Health Sci J 2014; 33:136-9.

4. Jo HS, Moon JY, Kim BG, Nam EW. Analysis of socio-demographics, self-rated health, social capital, and happiness in a medium-sized healthy city, Republic of Korea. J Lifestyle Med 2015; 5:68-75.

5. Weech-Maldonado R, Miller MJ, Lord JC. The relationships among socio-demographics, perceived health, and happiness. Appl Res Qual Life 2017; 12:289-302.

6. Ngamaba KH, Soni D. Are happiness and life satisfaction different across religious groups? Exploring determinants of happiness and life satisfaction. J Relig Health 2018; 57:2118-39.

7. Liu B, Floud S, Pirie K, Green J, Peto R, Beral $\mathrm{V}$, et al. Does happiness itself directly affect mortality? The prospective UK Million Women Study. Lancet 2016; 387:874-81.

8. Sato W, Kochiyama T, Uono S, Kubota Y, Sawada R, Yoshimura S, et al. The structural neural substrate of subjective happiness. Sci Rep 2015; 5:16891.

9. Sabatini F. The relationship between happiness and health: evidence from Italy. Soc Sci Med 2014; 114:178-87.

10. Diener E, Suh EM, Lucas RE, Smith HL. Subjective well-being: three decades of progress. Psychol Bull 1999; 125:276-302.

11. Rabito-Alcon MF, Rodriguez-Molina JM. Satisfaction with life and psychological well-being in people with gender dysphoria. Actas Esp Psiquiatr 2016; 44:47-54.

12. Pantic I. Online social networking and mental health. Cyberpsychol Behav Soc Netw 2014; 17:652-7.

13. Singer PW, Brooking ET. Like war: the weaponization of social media. New York: Eamon Dolan; 2018.

14. PNAD Contínua TIC 2017: Internet chega a três em cada quatro domicílios do país. Agência IBGE Notícias 2018; 20 dec. https://agen ciadenoticias.ibge.gov.br/agencia-sala-de-im prensa/2013-agencia-de-noticias/releases/ 23445-pnad-continua-tic-2017-internet-che ga-a-tres-em-cada-quatro-domicilios-do-pais.

15. Instituto Brasileiro de Geografia e Estatística. Acesso à Internet e à televisão e posse de telefone móvel celular para uso pessoal 2018. https://biblioteca.ibge.gov.br/visualizacao/li vros/liv101705_informativo.pdf (accessed on 15/Jul/2018).
16. We Are Social. Digital In 2019. https://weare social.com/global-digital-report-2019 (accessed on $15 / \mathrm{Jul} / 2019$ ).

17. Hervas G, Vazquez C. Construction and validation of a measure of integrative well-being in seven languages: the Pemberton Happiness Index. Health Qual Life Outcomes 2013; 11:66.

18. Paiva BS, de Camargos MG, Demarzo MM, Hervas G, Vazquez C, Paiva CE. The Pemberton Happiness Index: validation of the universal Portuguese version in a large Brazilian sample. Medicine (Baltimore) 2016; 95:e4915.

19. Gouveia VV, Barbosa GA, Andrade EO, Carneiro MB. Medindo a satisfação com a vida dos médicos no Brasil. J Bras Psiquiatr 2005; 54:298-305.

20. Pavot W, Diener E, Colvin CR, Sandvik E. Further validation of the Satisfaction with Life Scale: evidence for the cross-method convergence of well-being measures. J Pers Assess 1991; 57:149-61.

21. Hutz CS. Avaliação em psicologia positiva. Porto Alegre: Artmed; 2014.

22. Magidson J. SPSS for Windows: CHAID, release 6.0. Chicago: SPSS Inc.; 1993.

23. Della Giusta M, Jewell SL, Kambhampati US. Gender and life satisfaction in the UK. Fem Econ 2011; 17:1-34.

24. Blanchflower DG, Oswald AJ. Is well-being Ushaped over the life cycle? Soc Sci Med 2008; 66:1733-49.

25. Satuf C, Monteiro S, Pereira H, Esgalhado G, Marina Afonso R, Loureiro M. The protective effect of job satisfaction in health, happiness, well-being and self-esteem. Int J Occup Saf Ergon 2018; 24:181-9.

26. Russell JE. Promoting subjective well-being at work. J Career Assess 2008; 16:117-31.

27. Dulin PL, Gavala J, Stephens C, Kostick M, McDonald J. Volunteering predicts happiness among older Maori and non-Maori in the New Zealand health, work, and retirement longitudinal study. Aging Ment Health 2012; 16:617-24.

28. Huang LH. Well-being and volunteering: evidence from aging societies in Asia. Soc Sci Med 2018; 229:172-80.

29. Morrow-Howell N, Hong SI, Tang F. Who benefits from volunteering? Variations in perceived benefits. Gerontologist 2009; 49:91-102.

30. Pressman SD, Matthews KA, Cohen S, Martire LM, Scheier M, Baum A, et al. Association of enjoyable leisure activities with psychological and physical well-being. Psychosom Med 2009; 71:725-32.

31. Hart EAC, Lakerveld J, McKee M, Oppert JM, Rutter H, Charreire H, et al. Contextual correlates of happiness in European adults. PLoS One 2018; 13:e0190387. 
32. Adams KB, Leibbrandt S, Moon H. A critical review of the literature on social and leisure activity and wellbeing in later life. Ageing Soc 2011; 31:683-712.

33. Takeda F, Noguchi H, Monma T, Tamiya N. How possibly do leisure and social activities impact mental health of middle-aged adults in Japan? An evidence from a national longitudinal survey. PLoS One 2015; 10:e0139777.

34. Chiang HH, Lee TS. Family relations, sense of coherence, happiness and perceived health in retired Taiwanese: analysis of a conceptual model. Geriatr Gerontol Int 2018; 18:154-60.

35. Lobos G, Lapo MC, Schnettler B. In the choice between health and money, health comes first: an analysis of happiness among rural Chilean elderly. Cad Saúde Pública 2016; 32:e0032215.

36. Vázquez JJ. Happiness among the garbage: differences in overall happiness among trash pickers in León (Nicaragua). J Posit Psychol 2013; 8:1-11.

37. Adesanya AO, Rojas BM, Darboe A, Beogo I. Socioeconomic differential in self-assessment of health and happiness in 5 African countries: finding from World Value Survey. PLoS One 2017; 12:e0188281.

38. Biswas-Diener R, Diener E. Will money increase subjective well-being? A literature review and guide to needed research. Soc Indic Res 2001; 57:119-69.
39. North RJ, Holahan CJ, Moos RH, Cronkite RC. Family support, family income, and happiness: a 10-year perspective. J Fam Psychol 2008; 22:475-83.

40. Gilovich T, Kumar A. We'll always have Paris: the hedonic payoff from experiential and material investments. Adv Exp Soc Psychol 2015; 51:147-87.

41. Lathia N, Sandstrom GM, Mascolo C, Rentfrow PJ. Happier people live more active lives: using smartphones to link happiness and physical activity. PLoS One 2017; 12:e0160589.

42. Kanning M, Schlicht W. Be active and become happy: an ecological momentary assessment of physical activity and mood. J Sport Exerc Psychol 2010; 32:253-61.

43. Touburg G, Veenhoven R. Mental health care and average happiness: strong effect in developed nations. Adm Policy Ment Health 2015; 42:394-404.

44. Lombardo P, Jones W, Wang L, Shen X, Goldner EM. The fundamental association between mental health and life satisfaction: results from successive waves of a Canadian national survey. BMC Public Health 2018; 18:342.

45. Steptoe A. Happiness and health. Annu Rev Public Health 2019; 40:339-59.

46. Helliwell JF, Layard R, Sachs JD. World happiness report 2019. New York: Sustainable Development Solutions Network; 2019. 


\section{Resumo}

O estudo teve como objetivo identificar possíveis condições associadas com a percepção de felicidade e satisfação de vida em uma amostra de indivíduos da população geral brasileira que usam redes sociais. $O$ estudo transversal recrutou participantes via redes sociais nas cinco macrorregiões brasileiras, através do Facebook e do WhatsApp. Os dados foram coletados entre outubro de 2015 e outubro de 2016. Os instrumentos utilizados foram o Índice de Felicidade de Pemberton, Escala de Satisfação com a Vida e um questionário sobre características sociodemográficas e clinicas e questões potencialmente associadas com a sensação de felicidade. Foram incluídos 2.151 participantes. Cinco variáveis tiveram maior influência sob níveis mais altos de felicidade e de satisfação com a vida, de acordo com o modelo de regressão linear múltipla, análise de regressão logística multivariada e modelo de árvore de decisão. A satisfação com as circunstâncias financeiras, a autoavaliação da saúde positiva, reuniões familiares frequentes, atividade física $\geq 3$ vezes por semana $e$ ausência de diagnóstico psicológico ou psiquiátrico prévio são variáveis que "parecem" influenciar positivamente a percepção de felicidade e satisfação com a vida entre os brasileiros. Foram identificados alguns preditores de felicidade e satisfação com a vida, relacionados principalmente a atividades sociais e à satisfação pessoal. Uma medida útil nesse contexto é incentivar as pessoas a buscarem estratégias para elevar os níveis de felicidade $e$ de satisfação com a vida baseadas nas variáveis modificáveis identificadas no estudo.

Felicidade; Satisfação Pessoal; Rede Social

\section{Resumen}

El objetivo fue identificar posibles condiciones asociadas con la percepción de felicidad y satisfacción con la vida, en una muestra de individuos de la población general brasileña que utilizó redes sociales. Se trata de un estudio transversal, con participantes reclutados en línea en cinco regiones de Brasil, a través del Facebook y del WhatsApp. Los datos fueron recogidos entre octubre 2015 y octubre 2016. Los instrumentos usados fueron: el Índice de Felicidad de Pemberton, la Escala de Satisfacción con la Vida, y un cuestionario respecto a las características sociodemográficas y clínicas, así como asuntos potencialmente asociados con el sentimiento de felicidad. Se incluyeron a un total de 2.151 participantes. Cinco variables ejercieron la influencia más significativa en los mayores niveles de felicidad y satisfacción con la vida en el modelo de regresión múltiple, en los análisis de regresión logística múltiple y en el modelo de árbol de decisión. Estar satisfecho con las circunstancias financieras, tener una autoevaluación positiva de salud, reunirse frecuentemente con la familia, implicarse en actividades físicas $\geq 3$ veces a la semana, y no contar diagnósticos previos psicológicos/psiquiátricos son variables que "parecen" influenciar positivamente la percepción de felicidad y satisfacción con la vida de los brasileños. Se identificaron algunos predictores de felicidad y satisfacción con la vida, lo que estaba principalmente relacionado con actividades sociales y satisfacción personal de los participantes brasileños. Fomentar que la gente busque estrategias para incrementar los niveles de felicidad y satisfacción con la vida, basados en variables modificables como las encontradas, puede ser útil en este contexto.

Felicidad; Satisfacción Personal; Red Social
Submitted on $22 /$ Jun/2020

Final version resubmitted on 19/Jan/2021

Approved on 04/Mar/2021 